\title{
Evaluation of Bi-Iliac Distance and Timing of Ossification of Sacrum by Sonography in the Second Trimester of Pregnancy
}

\author{
Behnaz Moradi ${ }^{1,2}$, Abbas Ghanbari ${ }^{3,}$, , Maryam Rahmani ${ }^{1}$, Mohamad Ali Kazemi ${ }^{4}$, Ahmad-Reza \\ Tahmasebpour ${ }^{5}$ and Madjid Shakiba ${ }^{1}$ \\ Advanced Diagnostic and Interventional Radiology Research Center, Imam Khomeini Hospital,Tehran University of Medical Sciences, Tehran, Iran \\ ${ }^{2}$ Department of Radiology, Women' Yas Hospital, Tehran University of Medical Science, Tehran, Iran \\ ${ }^{3}$ Department of Radiology, Imam Khomeini Hospital, Tehran University of Medical Sciences, Tehran, Iran \\ ${ }^{4}$ Department of Radiology, Amiralam Hospital, Tehran University of Medical Sciences, Tehran, Iran \\ ${ }^{5}$ Iranian Fetal Medicine Foundation, Hope Generation Foundation, Tehran, Iran \\ "Corresponding author: Department of Radiology, Imam Khomeini Hospital, Keshavarz Blvd., Tehran, Iran. Tel: +98-9123673295, Fax: +98-2166581577, Email: \\ dr.abbasghanbari@yahoo.com
}

Received 2018 May 29; Revised 2019 January 17; Accepted 2019 January 26.

\begin{abstract}
Background: The sacral region in the fetus can be involved in many pathologies such as spina bifida as the most common spinal abnormality and caudal regression syndrome. On this basis, knowledge about the temporal sequences of sacral ossification centers appearances and other characteristics including bi-iliac distance with newer higher resolution ultrasound technique may be helpful in defining pathologies involving the sacral region and fetal skeletal maturation.

Objectives: The goal of this research was to evaluate the timing of ossification of sacral vertebrae and bi-iliac distance as a guidance for clinicians to make an early and reliable diagnosis of sacral region anomalies such as caudal regression syndrome.

Patients and Methods: This cross-sectional study was done on 315 normal pregnancies at 14 to 25 weeks of gestational age. The sacral region was evaluated in spine up position, on a coronal plane. By changing the orientation of the probe between sagittal and coronal planes and continuous observation, presence of the ossification center could be confirmed. Then, the level of the central and neural arch centers was recorded in every gestational age.

Results: Ossification happened in a predictable caudal direction pattern. S1 and body of S2 were visualized in all cases at 15 - 17 weeks + 6 days and all fetuses with 17 weeks of gestational age. The body of S3 was detected in all cases at 17 weeks and its arch at 20 weeks. Studying all fetuses at 18 weeks of gestation shows sacral ossification in the body and arch of S4. During the study period we had two cases with sirenomelia and three cases with sacral agenesis and we have explained their sacral region findings.

Conclusion: By using recent high-resolution ultrasound machines, sacral vertebral ossification centers could be identified in its early gestational age, which could be helpful for earlier detection of sacral pathologies.
\end{abstract}

Keywords: Spine, Ossification Center, Ultrasound, Sacral Anomalies, Bi-iliac Distance

\section{Background}

These days an early detection of fetal structural abnormalities has become a main part of modern obstetrical care. The sacral region in the fetus could be involved in many pathologies such as spina bifida as the most common spinal abnormality and caudal regression syndrome which represents a spectrum of congenital defects ranging from isolated sacral agenesis to sirenomelia. As other examples, delayed ossification centers in osteochondrodysplasias and a small skeletal system in lethal skeletal dysplasia $(1,2)$ could be mentioned.

Ossification of sacral centers occur in a predictable pattern and are closely related to fetal gestational age. Timing of spinal ossification has been studied with histo- logic, radiographic, and ultrasound methods (1-6). Highresolution ultrasonography has an integral role in the evaluation of the ossified part in the fetal skeletal system (7).

On this basis, knowledge about temporal sequences of sacral ossification center appearance and other characteristics including the bi-iliac distance with newer higher resolution ultrasound technique may be helpful to define pathologies involving the sacral region and fetal skeletal maturation which is the aim of our study.

\section{Objectives}

The main goal of this research is to define a nomogram for sacral nuclei ossification in order to reach an earlier 
diagnosis of sacral anomalies including caudal regression syndrome. Another purpose was to measure the bi-iliac distance for each gestational week and for every gender as an adjunct index for evaluation of sacral region anomalies.

\section{Patients and Methods}

The present cross sectional study was performed from September 2015 to February 2017 at Yas women's hospital in Tehran. Our institutional ethics board approved this study and informed consent was obtained from all individual participants.

A total of 315 women were evaluated for detection of fetal structural abnormalities in the second trimester screening ultrasonography.

All participants were single pregnancy, with good US visualization and no history of bone dysplasia. Ten fetuses (3\%) were excluded due to poor visualization related to maternal body habitus. The exclusion criteria were presence of fetal anomalies during the study and an increased risk of first trimester screening at 11 to 14 weeks of gestation associated with fetal weight less than $10 \%$, maternal diabetes and drug ingestion. In addition, fetuses with any abnormality in follow-up sonography or after birth were excluded from the study.

Gestational age was calculated based on the crownrump length $(\mathrm{CRL})$ evaluated in the first trimester sonography between 10 weeks and 13 weeks and 6 days and its range differed from 14 weeks to 25 weeks and 6 days.

The sacral region was evaluated in spine up position on a coronal plane. Based on a study performed by Budorick et al. in 1991, the first sacral vertebrae was at the level of the upper iliac contour and the distal sacral ossification centers could easily be assessed (3).

Magnification of the image was such that the fetal sacrum occupied half of the image. Changing probe orientation between sagittal and coronal planes during continuous viewing was carried out to confirm the presence of the ossification center. Then, the level of central and neural arch ossification centers of sacral vertebrae was recorded in each fetus (Figure 1A).

The inner to inner distance between the two iliac wings was also measured in its upper and lower contours in the same plane and magnification used for sacral ossification counting. The distance was measured three times by the same operator for each fetus and the average of these recorded measurements was finally considered as the true value. These distances were adequately recorded in all fetuses and compared with the counting ossification centers, they were less limited by maternal obesity (Figure 1B). The number of ossification nuclei and bi-iliac distances were recorded adequately in all 315 fetuses.

During the study period we had two cases with sirenomelia and three cases with sacral agenesis and we
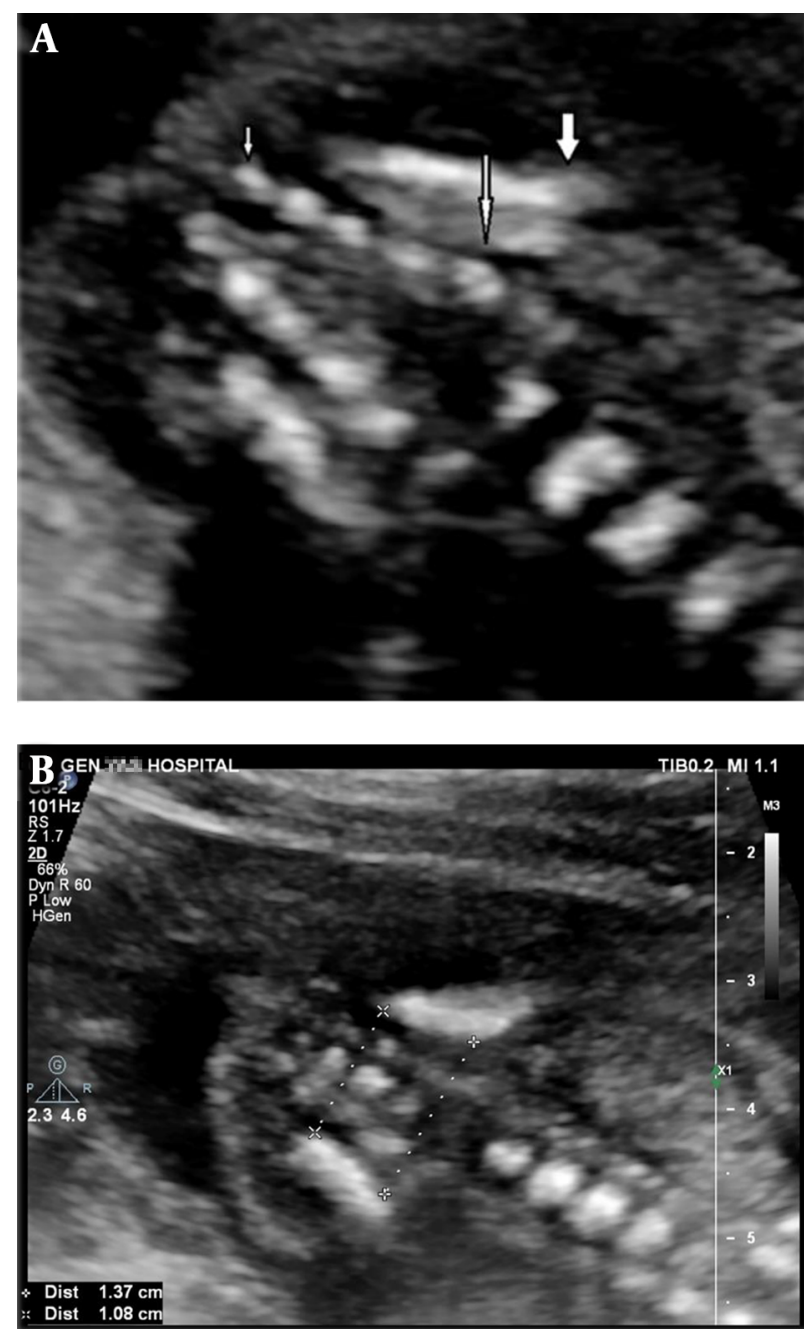

Figure 1. A, Ultrasound scan at 19 weeks of gestation in the coronal section in spine up position shows 5 sacral ossification nuclei. Thick arrow: upper contour of iliac wing; large thin arrow: S1; small thin arrow: S5; B, Method of bi-iliac distance measurement in the same section in another 19 weeks gestation fetus

had explained their sacral region findings and especially compared sacral sonographic findings of sacral agenesis cases with the results of normal fetuses.

Measurements were carried out in all fetuses by a trans-abdominal convex array transducer with $2-6 \mathrm{MHz}$ frequency (Affinity 50, General imaging configuration, Philips ultrasound machine, USA).

All the statistical tests were two-sided; a p value of less than 0.05 was considered statistically significant. All the analyses were done by statistical software SPSS version 22 (IBM Corp. released 2013. Armonk, NY). 


\section{Results}

Totally, 315 women were included in this study. The mean age of women was $29.9 \pm 5.1$ years (Range: 18 - 44). One hundred seventy-two subjects (54.4\%) were gravid one, 91(28.8\%) were gravid two and the rest of participants were gravid three or more. One hundred sixty-one (50.9\%) of all fetuses were female and 155 (49.1\%) were male (Table 1).

\begin{tabular}{|c|c|}
\hline Variables & Values \\
\hline \multicolumn{2}{|l|}{ Age } \\
\hline Mean \pm SD & $29.9 \pm 5.1$ \\
\hline Range & $18-44$ \\
\hline Inter-quartile range & 8 \\
\hline \multicolumn{2}{|l|}{ Gravidity } \\
\hline 1 & $172(54.4)$ \\
\hline 2 & $91(28.8)$ \\
\hline 3 & $45(14.2)$ \\
\hline 4 & $8(2.5)$ \\
\hline \multicolumn{2}{|l|}{ Parity } \\
\hline 0 & $183(57.9)$ \\
\hline 1 & $103(32.6)$ \\
\hline 2 & $27(8.5)$ \\
\hline 3 & $3(0.9)$ \\
\hline \multicolumn{2}{|l|}{ Fetal gender } \\
\hline Male & $155(49.1)$ \\
\hline Female & $161(50.9)$ \\
\hline
\end{tabular}

Abbreviation: SD, standard deviation

${ }^{\text {a }}$ Values are expressed as No. (\%) unless otherwise indicated.

The number of ossified bodies, neural arch nuclei and bilateral iliac distance of each fetus was separately recorded for every week of gestation. Table 2 demonstrates the number of cases and the percentage of ossified nuclei for each gestational age between 14 to 25 weeks in all fetuses.

S1 and body of S2 were visualized in all cases at 15 - 17 weeks +6 days and the arch of S2 in all fetuses with 17 weeks of gestational age. The body of S3 was detected in all cases at 17 weeks and its arch at 20 weeks. Sacral ossification was depicted in the body and arch of S4 in all fetuses at 20 weeks of gestational age. Ossification of S5 was seen at 18 weeks and with a higher percentage during the following weeks of gestation. As evident in the Tables, neural arch ossification centers are visualized later.

Distribution of the starting level of ossification in each gestational week did not show any statistical difference between male and female fetuses for the body and arch of the sacral vertebrae (all P-values were greater than 0.19 and most of them were greater than 0.45). Similar patterns were seen for different groups of fetuses classified based on gravidity and parity (all P-values were greater than 0.07 ; most of them were greater than 0.35 ).

The bi-iliac distance for each gestational week is summarized as a nomogram in Table 3. The percentiles for each gestational age are presented for the distance of the upper and lower contour of bilateral iliac bones.

Figures 2 and 3 show scatter diagrams of bi-iliac distance plotted on 5th and 95th percentiles in the upper and lower iliac contours. The diagrams present the gestational age of the growths, distances which are continuously increased in a predictive manner. Like ossification timing and pattern of sacral nuclei, fetal gender and maternal gravidity has no statistically significant effect on bi-iliac distances.

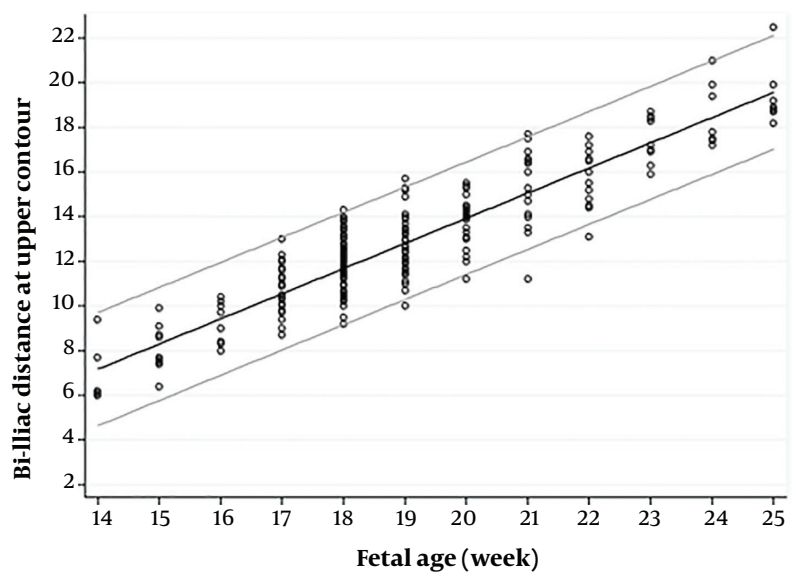

Figure 2. Scatter diagram of bi-iliac distance plotted on 5th and 95th percentiles in the upper iliac contour

During the study period, five cases with sacral pathologies excluding neural tube defect cases were also referred to our hospital, including two cases with sirenomelia, and three cases with sacral agenesis (Table 4 and Figure 4).

We excluded spina bifida cases because they are usually diagnosed based on more other obvious findings such as cranial signs. Both of our sirenomelia cases were mostly severe forms, only a single bone was present, with no legs or feet (Type 7 based on Stocker and Heifetz classification). In these two cases, sacral agenesis was identified with close contact of both iliac wings. In one of them, all central and lateral lumbar centers showed the ossification with associated blocked vertebrae (case 1). In another case (case 2) the last ossified vertebral center was L4 in the arch and L5 in the body and it was associated with hemivertebra and mild scoliosis.

One of the cases (case 3) with sacral agenesis was 18 
Moradi B et al.

\begin{tabular}{|c|c|c|c|c|c|c|c|c|c|c|c|}
\hline \multirow{2}{*}{$\begin{array}{l}\text { Gestational } \\
\text { age (week- } \\
\text { week+day) }\end{array}$} & \multirow{2}{*}{$\begin{array}{c}\text { Number } \\
\text { of cases }\end{array}$} & \multicolumn{2}{|c|}{ S1 } & \multicolumn{2}{|c|}{ S2 } & \multicolumn{2}{|c|}{ S3 } & \multicolumn{2}{|c|}{ S4 } & \multicolumn{2}{|c|}{ S5 } \\
\hline & & Body & Arch & Body & Arch & Body & Arch & Body & Arch & Body & Arch \\
\hline $14-14+6$ & 6 & $5(83)$ & $5(83)$ & $5(83)$ & $3(50)$ & $3(50)$ & 0 & 0 & 0 & 0 & 0 \\
\hline $15-15+6$ & 9 & $9(100)$ & $9(100)$ & $9(100)$ & $6(66)$ & $6(66)$ & 0 & 0 & 0 & 0 & 0 \\
\hline $16-16+6$ & 9 & $9(100)$ & $9(100)$ & $9(100)$ & $6(66)$ & $7(77)$ & $2(22)$ & $1(11)$ & $1(11)$ & 0 & 0 \\
\hline $17-17+6$ & 28 & $28(100)$ & $28(100)$ & $28(100)$ & $28(100)$ & $28(100)$ & $16(57)$ & $19(67)$ & $5(18)$ & 0 & 0 \\
\hline $18-18+6$ & 96 & $96(100)$ & $96(100)$ & $96(100)$ & $96(100)$ & $96(100)$ & $79(82)$ & $92(96)$ & $56(57)$ & $4(4.2)$ & $2(2.1)$ \\
\hline $19-19+6$ & 72 & $72(100)$ & $72(100)$ & $72(100)$ & $72(100)$ & $72(100)$ & $68(94.5)$ & $71(98.5)$ & $59(80)$ & $5(7)$ & $4(4)$ \\
\hline $20-20+6$ & 35 & $35(100)$ & $35(100)$ & $35(100)$ & $35(100)$ & $35(100)$ & $35(100)$ & $35(100)$ & $35(100)$ & $2(5.7)$ & $2(5.7)$ \\
\hline $21-21+6$ & 23 & $23(100)$ & $23(100)$ & $23(100)$ & $23(100)$ & $23(100)$ & $23(100)$ & $23(100)$ & $23(100)$ & $6(26)$ & $5(21)$ \\
\hline $22-22+6$ & 13 & $13(100)$ & $13(100)$ & $13(100)$ & $13(100)$ & $13(100)$ & $13(100)$ & $13(100)$ & $13(100)$ & $5(38)$ & $4(30)$ \\
\hline $23-23+6$ & 9 & $9(100)$ & $9(100)$ & $9(100)$ & $9(100)$ & $9(100)$ & $9(100)$ & $9(100)$ & $9(100)$ & $2(22)$ & $1(11)$ \\
\hline $24-24+6$ & 8 & $8(100)$ & $8(100)$ & $8(100)$ & $8(100)$ & $8(100)$ & $8(100)$ & $8(100)$ & $8(100)$ & $5(62)$ & $3(37)$ \\
\hline $25-25+6$ & 7 & $7(100)$ & $7(100)$ & $7(100)$ & $7(100)$ & $7(100)$ & $7(100)$ & $7(100)$ & $7(100)$ & $7(100)$ & $3(42)$ \\
\hline
\end{tabular}

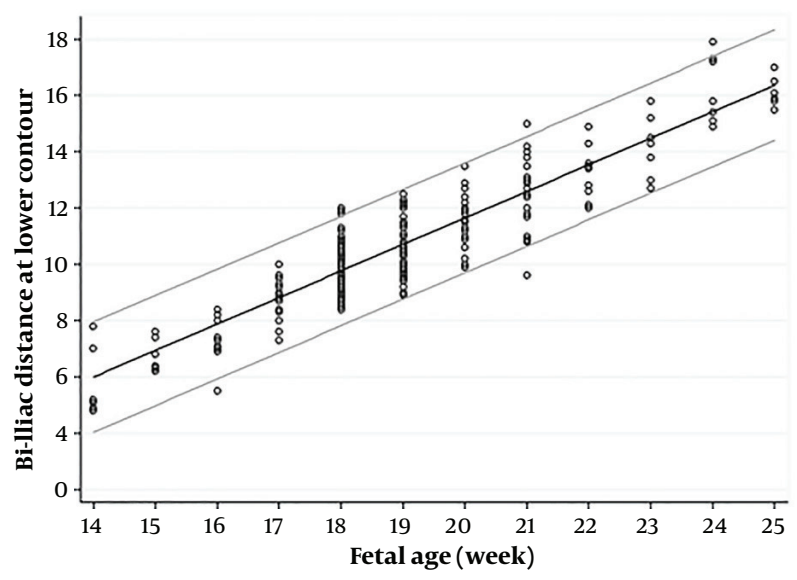

Figure 3. Scatter diagram of bi-iliac distance plotted on 5th and 95th percentiles in the lower iliac contour

weeks + 3 days and the last ossification center was L3 in the arch and L5 in the body. Bodies of L4 and L5 were also fused together. Both iliac wings articulated with the last vertebra and the bi-iliac distance were very small, approximately 4.5 $\mathrm{mm}$ and $2.6 \mathrm{~mm}$ for the upper and lower contours, respectively (type 3 based on Renshaw classification). Another case of sacral agenesis (case 4 ) was 18 weeks +2 days. The last ossified vertebral centers were $\mathrm{S} 1$ in the arch and S2 in the body and the bi-iliac distance was also decreased; 9.8 $\mathrm{mm}$ and $5.7 \mathrm{~mm}$ for the upper and lower contours, respectively, which were under the 5th percentile based on our result (probably type 2 ). The last case was referred for corpus callosum agenesis and omphalocele at 17 weeks and we identified diastematomyelia with bony spur at the L5 level. In addition, we noted a unilateral partial agenesis on the left side. On the left side of the sacrum, two lateral ossification centers were evident and on the right side, four ossification nuclei were detected. Bi-iliac distances were $10 \mathrm{~mm}$ (between percentile 25th and 50th) at the upper contour and $7 \mathrm{~mm}$ (less than 5 th percentile) in the lower part in favor of type 1 . The widening effect of diastematomyelia at the upper contour of the iliac wings might possibly result in increased distances at the upper part which would put it in the normal range.

\section{Discussion}

Knowledge of the timing and pattern of ossification could be helpful in diagnosing and defining the possible structural abnormalities of the distal spine including spina bifida, impaired ossification of the vertebra and caudal regression syndrome spectrum, especially for the mild form of isolated sacral agenesis as two of our cases. So, it becomes important in the mid second trimester when radiologists most frequently ask to exclude fetal anomalies. For instance, caudal regression syndrome with isolated lumbosacral agenesis which is not a rare condition in the setting of diabetic mothers becomes suspicious based on the shortened spinal length at 12 weeks, and it may be confirmed by the absence of sacral ossification centers at 16 weeks (1).

Based on the previous performed studies, ossification of the fetal spine has a predictable timing and pattern. The bodies start to ossify from T10 - L1 vertebra and the ossification progresses in both cephalad and caudal directions. 


\begin{tabular}{|c|c|c|c|c|c|c|c|c|}
\hline \multirow{2}{*}{ Gestational weeks } & \multirow{2}{*}{ Mean \pm SD } & \multirow{2}{*}{ IQR } & \multicolumn{5}{|c|}{ Percentiles } & \multirow{2}{*}{ Range } \\
\hline & & & 5th & 25th & 5oth & 75th & 95th & \\
\hline \multicolumn{9}{|l|}{$14 w-14 w+6 d(n=6)$} \\
\hline Upper contour & $6.9 \pm 1.39$ & 2.13 & 6 & 6 & 6.15 & 8.13 & 9.4 & $6-9.4$ \\
\hline Lower contour & $5.8 \pm 1.27$ & 2.33 & 4.8 & 4.89 & 5.15 & 7.2 & 7.8 & $4.8-7.8$ \\
\hline \multicolumn{9}{|l|}{$15 w-15 w+6 d(n=9)$} \\
\hline Upper contour & $8.1 \pm 1.06$ & 1.45 & 6.4 & 7.45 & 7.7 & 8.9 & 9.9 & $6.4-9.9$ \\
\hline Lower contour & $6.73 \pm 0.58$ & 1.15 & 6.2 & 6.25 & 6.4 & 7.4 & 7.6 & $6.2-7.6$ \\
\hline \multicolumn{9}{|l|}{$16 w-16+6 d(n=9)$} \\
\hline Upper contour & $9.11 \pm 0.98$ & 1.95 & 8 & 8.15 & 9 & 10.1 & 10.4 & $8-10.4$ \\
\hline Lower contour & $7.31 \pm 0.87$ & 1.15 & 5.5 & 6.95 & 7.3 & 8.1 & 8.4 & $5.5-8.4$ \\
\hline \multicolumn{9}{|l|}{$17 w-17 w+6 d(n=28)$} \\
\hline Upper contour & $10.62 \pm 1.05$ & 1.42 & 8.84 & 9.85 & 10.5 & 11.28 & 12.69 & $8.7-13$ \\
\hline Lower contour & $8.96 \pm 0.65$ & 0.8 & 7.44 & 8.7 & 9 & 9.5 & 10 & $7.3-10$ \\
\hline \multicolumn{9}{|l|}{$18 w-18 w+6 d(n=97)$} \\
\hline Upper contour & $11.84 \pm 1.45$ & 1.45 & 10 & 11.3 & 11.7 & 12.75 & 14 & $2.6-14.3$ \\
\hline Lower contour & $9.92 \pm 1.11$ & 1.15 & 8.59 & 9.35 & 10 & 10.5 & 11.35 & $2.5-12$ \\
\hline \multicolumn{9}{|l|}{$19 w-19 w+6 d(n=72)$} \\
\hline Upper contour & $12.73 \pm 1.15$ & 1.38 & 10.90 & 12 & 12.9 & 13.38 & 15 & $10-15.7$ \\
\hline Lower contour & $10.71 \pm 0.88$ & 1.30 & 9.33 & 10 & 10.65 & 11.3 & 12.24 & $8.9-12.5$ \\
\hline \multicolumn{9}{|l|}{$20-20+6 d(n=35)$} \\
\hline Upper contour & $13.96 \pm 1.11$ & 2 & 11.84 & 13 & 14.2 & 15 & 15.42 & $11.2-15.5$ \\
\hline Lower contour & $11.63 \pm 0.9$ & 1.20 & 9.98 & 11 & 11.6 & 12.2 & 13.02 & $9.9-13.5$ \\
\hline \multicolumn{9}{|l|}{$21 w-21 w+6 d(n=23)$} \\
\hline Upper contour & $15.3 \pm 1.56$ & 2.5 & 11.62 & 14.1 & 15.3 & 16.6 & 17.66 & $11.2-17.7$ \\
\hline Lower contour & $12.49 \pm 1.3$ & 1.80 & 9.84 & 11.7 & 12.5 & 13.5 & 14.84 & $9.69-15$ \\
\hline \multicolumn{9}{|l|}{$22 w-22 w+6 d(n=13)$} \\
\hline Upper contour & $15.72 \pm 1.29$ & 2.1 & 13.1 & 14.65 & 16 & 16.75 & 17.6 & $13.1-17.6$ \\
\hline Lower contour & $13.19 \pm 0.86$ & 1.20 & 12 & 12.35 & 13.4 & 13.55 & 14.9 & $12-14.9$ \\
\hline \multicolumn{9}{|l|}{$23 w-23 w+6 d(n=9)$} \\
\hline Upper contour & $17.47 \pm 1.04$ & 1.85 & 15.9 & 16.6 & 17.2 & 18.45 & 18.7 & $15.9-18.7$ \\
\hline Lower contour & $14.33 \pm 1.03$ & 1.80 & 12.7 & 13.4 & 14.5 & 15.2 & 15.8 & $12.7-15.8$ \\
\hline \multicolumn{9}{|l|}{$24 w-24 w+6 d(n=8)$} \\
\hline Upper contour & $18.45 \pm 1.44$ & 2.38 & 17.2 & 17.4 & 17.65 & 19.78 & 21 & $17.2-21$ \\
\hline Lower contour & $16.18 \pm 1.13$ & 2.10 & 14.9 & 15.18 & 15.8 & 17.28 & 17.9 & $14.9-17.9$ \\
\hline \multicolumn{9}{|l|}{$25 w-25 w+6 d(n=7)$} \\
\hline Upper contour & $19.46 \pm 1.44$ & 1.2 & 18.2 & 18.7 & 18.9 & 19.9 & 22.5 & $18.2-22.5$ \\
\hline Lower contour & $16.1 \pm 0.5$ & 0.7 & 15.5 & 15.8 & 15.9 & 16.5 & 17 & $15.5-17$ \\
\hline
\end{tabular}

Abbreviation: d, day; IQR, interquartile range; SD, standard deviation; w, week. 


\begin{tabular}{|c|c|c|c|c|c|c|c|}
\hline Case & MA & Clinical history & GA & Fetal gender & Associated US finding & Outcome & Confirmation \\
\hline 1-Sirenomelia & 25 & - & $17 w+4 d$ & $?$ & $\begin{array}{l}\text { SUA, bilateral renal agenesis, } \\
\text { dilated cardiomyopathy, } \\
\text { pericardial effusion, severe } \\
\text { oligohydramnios, blocked } \\
\text { vertebrae }\end{array}$ & TOP & External examination \\
\hline 2-Sirenomelia & 28 & - & $17 \mathrm{w}$ & $?$ & $\begin{array}{l}\text { SUA, bilateral renal cystic } \\
\text { disease, hemivertebra and mild } \\
\text { scoliosis, mild ventriculomegaly, } \\
\text { severe oligohydramnios }\end{array}$ & TOP & External examination \\
\hline 3-Sacral agenesis & 35 & DM, IVF & $18 w+3 d$ & $\mathrm{~F}$ & $\begin{array}{l}\text { Decreased fetal feet movement, } \\
\text { Unilateral club foot, Soft tissue } \\
\text { wasting in lower limbs }\end{array}$ & TOP & External examination \& graphy \\
\hline 4-Sacral agenesis & 28 & - & $18 w+2 d$ & M & Unilateral club foot & TOP & Not performed \\
\hline 5-Sacral agenesis & 30 & DM & $17 \mathrm{w}$ & $\mathrm{F}$ & $\begin{array}{l}\text { Corpus callosum agenesis, } \\
\text { omphalocele, sacral } \\
\text { diastematomyelia, blocked } \\
\text { vertebrae in sacrum, soft tissue } \\
\text { wasting in lower limbs }\end{array}$ & TOP & $\begin{array}{l}\text { External examination and } \\
\text { graphy }\end{array}$ \\
\hline
\end{tabular}

Abbreviations: d, day; DM, diabetes mellitus; F, female; GA, gestational age; IVF, in vitro fertilization; M, male; MA, maternal age; SUA, single umbilical artery; TOP, termination of pregnancy; US, ultrasonography; w, week.
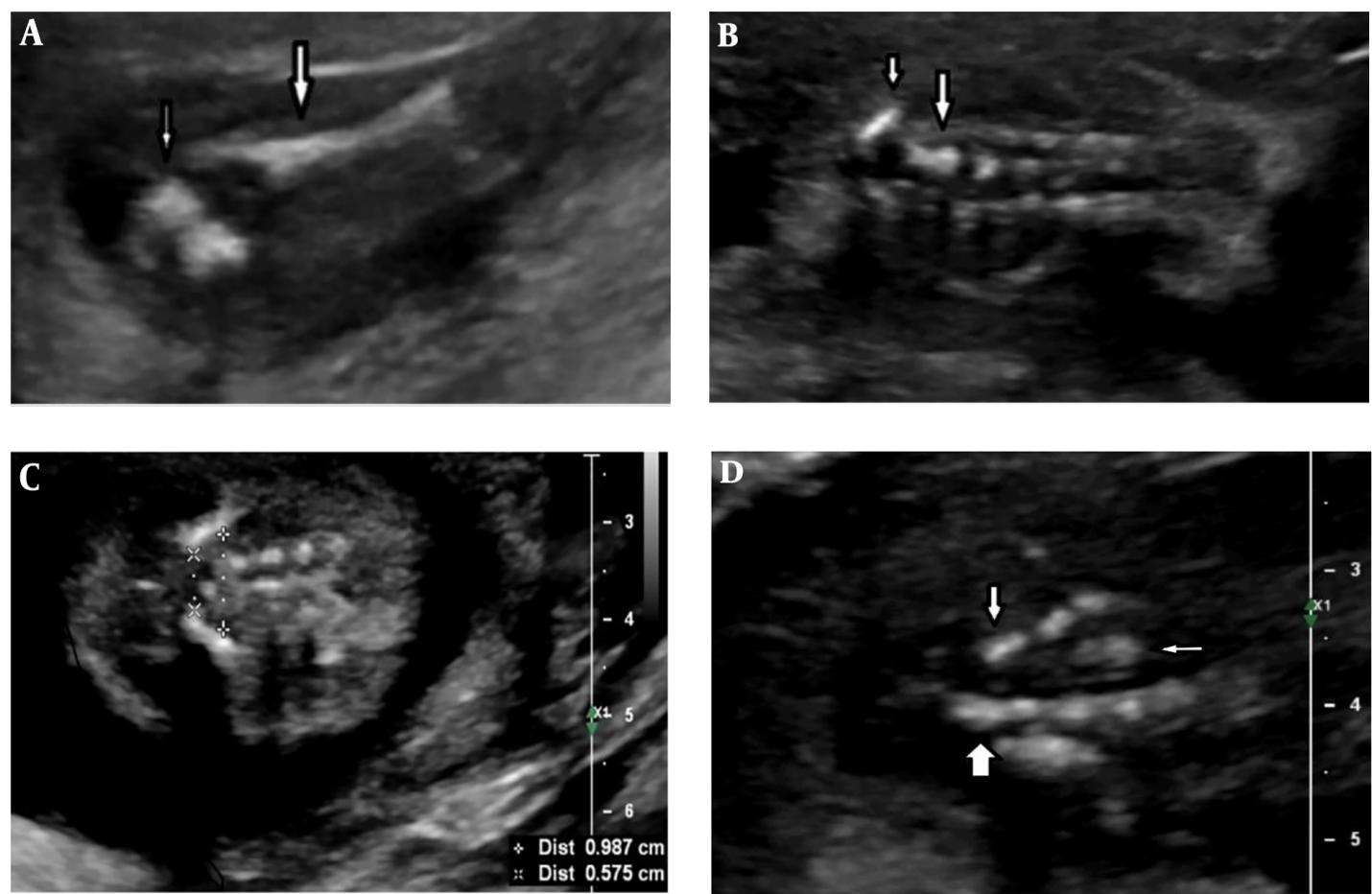

Figure 4. A, A 17 weeks + 4 days case of sirenomelia (case 1) with sacral agenesis and close contact of both iliac wings (small arrow) and only a single bone was present (large arrow), with no legs or feet; B, In case 3 with sacral agenesis, the last ossification center was L3 in the arch and L5 in the body. Bodies of L4 and L5 are fused together (large arrow). Both iliac wings articulated with the last vertebra and bi-iliac distance were very small especially in the lower contour (small arrow); C, Case 4 with mild sacral agenesis had small bi-iliac distance ( $9.8 \mathrm{~mm}$ and $5.7 \mathrm{~mm}$ for upper and lower contours, respectively) under the 5th percentile based on our result; D, Case 5 with diastematomyelia (horizontal arrow) and partial unilateral sacral agenesis with two ossification centers on the left side (small vertical arrow: S2) and four ossification nuclei on the other side with blocked vertebra of S3 and S4 (wide vertical arrow). The fetus also had a small bi-iliac distance in the lower contour ( $7 \mathrm{~mm}$ ) and normal in upper contours (10 mm).

As cephalad progression is quicker, neural arch nuclei ossification has different patterns, starting from the first cervical spine and making progress down to the sacral region
(8). Radiologic (9-11) and sonographic studies $(3,5,12)$ agree with this developmental pattern.

On the contrary, histologic investigations have shown 
different ossification temporal patterns. In other words, histologic studies show mineralization of the nuclei in earlier weeks compared to radiologic and sonographic studies $(1,13,14)$. Maybe because the mineralization should reach a level that is detectable by sonographic machines.

Bareggi et al. using the double staining technique at 10 to 16 weeks of gestation, showed that all sacral ossification nuclei (bodies and arches) were present at 16 weeks of gestation (8).

In this study, $S 1$ and the body of S2 were visualized in all cases at $15-17$ weeks +6 days and the arch of $S 2$ and body of S3 were detected in all cases at 17 weeks and the S3 arch at 20 weeks. Sacral ossification was depicted in the body and arch of S4 in all fetuses at 20 weeks of gestational age. In comparison with the study conducted by De Biasio et al. (1) and van Zalen-Sprock et al. (7), ossification centers in our study were identified earlier.

Ossification in S5 was first found at 18 weeks in this study, at 21 weeks in the study performed by De Biasio et al. and at 20 weeks in only female fetuses in a study carried out by Vignolo et al. However, this result could be partly related to different sample sizes which was higher in this study. The percentage of its appearance at 20 and 21 weeks is nearly similar to the mentioned investigations. In our study, the percentages were $26 \%$ in the body and $21 \%$ in the arch. In the study performed by De Biasio et al. it was $27 \%$ in the body and $21 \%$ in the arch, and in a study done by Vignolo et al., it was $42 \%$ in the body and $28 \%$ in the arch in the female group.

In this study, S2 body nucleus was detected in all male fetuses one week earlier and S3 body nucleus 4 weeks earlier than the study conducted by Vignolo et al. (2005). On the other hand, no obvious difference was found between the two studies regarding female fetuses. This result reflects that the percentage of appearance of sacral vertebrae ossification centers in male fetuses was lower compared to this study (4).

High-resolution ultrasonographic machines make it possible to visualize ossification centers earlier than past generation machines, however with respect to histology, visualization of ossification is still later.

We measured the bi-iliac distance at the upper and lower iliac contour in the same plane used to assess the sacrum (Table 3). The results show the distance at the upper portion is always greater than the distal bi-iliac distance, and as the fetus is growing, the distance will increase in a predictable and linear pattern. These distances decreased in all our sacral agenesis (much more in case 3 ) and could be helpful in better understanding the sacral pathology along with ossification centers. Nevertheless, routine measurement of bi-iliac distance is not needed. However, if there is any doubt about existence of mild forms of sacral agenesis (type 1 and 2), bi-iliac distance measurement is a very helpful additional option similar to our case 4 and 5 .
Bi-iliac distance is also increased in spina bifida of the sacrum, and may be helpful especially in its mild forms. So, for the first time we propose bi-iliac distance as a contributory index along with counting ossified nuclei for identifying sacral pathology.

By using them, not only mild pathologies could be identified, but also the severity of sacral and lower lumbar vertebral involvement could be investigated. However, additional investigations need to confirm its practical role.

Previous studies have shown that ossification centers appear earlier in female fetuses $(1,4,15)$ but our study showed fetal gender has no obvious effect on ossification timing and pattern; as well as on bi-iliac distance.

One of the major advantages of this study was having a larger sample volume in comparison with previous studies [De Biasio et al. (1), Vignolo et al. (4)] that leads to an improved and accurate finding with the least of accidental errors. Another advantage was the application of a newer high resolution ultrasound machine which helped to detect the ossification nuclei in early gestational age.

In conclusion, the presented data support the previous pattern of craniocaudal sacral ossification. By using recent high-resolution ultrasound machines, the sacral vertebral ossification centers can be identified in early gestational age which helps to detect lower spine pathologies sooner. Furthermore, our study introduces a new index (biiliac distance) for evaluating the fetal sacrum.

\section{Footnotes}

Authors' Contributions: Study concept and design: Behnaz Moradi; acquisition of data: Behnaz Moradi and Abbas Ghanbari; analysis and interpretation of data: Madjid Shakiba; critical revision of the manuscript for the important intellectual content: Maryam Rahmani, Mohamad Ali Kazemi, Ahmad Reza Tahmasebpour; drafting of the manuscript: Abbas Ghanbari; statistical analysis: Madjid Shakiba

Conflict of Interests: None declared.

Ethical Approval: IR.TUMS.MEDICINE.REC.1395.1099.

Financial Disclosure: None declared.

Funding/Support: The authors declare that no grants or funds were received.

\section{References}

1. De Biasio P, Ginocchio G, Vignolo M, Ravera G, Venturini PL, Aicardi G. Spine length measurement in the first trimester of pregnancy. Prenat Diagn. 2002;22(9):818-22. doi:10.1002/pd.428. [PubMed: 12224078].

2. Szpinda M, Szpinda A, Flisinski P, Flisinski P. The normal growth of cross-sectional areas of the aorto-iliac segment in human fetuses - an anatomical, digital, and statistical study. Med Sci Monit Basic Res. 2013;19:46-53. doi: 10.12659/MSMBR.883756. [PubMed: 23370918]. [PubMed Central: PMC3638685]. 
3. Budorick NE, Pretorius DH, Grafe MR, Lou KV. Ossification of the fetal spine. Radiology.1991;181(2):561-5. doi:10.1148/radiology.181.2.1924805. [PubMed: 1924805].

4. Vignolo M, Ginocchio G, Parodi A, Torrisi C, Pistorio A, Venturini PL, et al. Fetal spine ossification: The gender and individual differences illustrated by ultrasonography. Ultrasound Med Biol. 2005;31(6):733-8. doi: 10.1016/j.ultrasmedbio.2005.02.013. [PubMed: 15936488].

5. Filly RA, Simpson GF, Linkowski G. Fetal spine morphology and maturation during the second trimester. Sonographic evaluation.J Ultrasound Med.1987;6(11):631-6. [PubMed: 3316699].

6. Bagnall KM, Harris PF, Jones PR. A radiographic study of the human fetal spine. 2 . The sequence of development of ossification centres in the vertebral column. J Anat. 1977;124(Pt 3):791-802. [PubMed: 604345]. [PubMed Central: PMC1234674].

7. van Zalen-Sprock RM, Brons JT, van Vugt JM, van der Harten HJ, van Geijn HP. Ultrasonographic and radiologic visualization of the developing embryonic skeleton. Ultrasound Obstet Gynecol.1997;9(6):392-7. doi: 10.1046/j.1469-0705.1997.09060392.x. [PubMed: 9239824].

8. Bareggi R, Grill V, Zweyer M, Narducci P, Forabosco A. A quantitative study on the spatial and temporal ossification patterns of vertebral centra and neural arches and their relationship to the fetal age. Ann Anat Anat Anzeig. 1994;176(4):311-7. doi: 10.1016/s0940-9602(11)80502-

9. O'Rahilly R, Muller F, Meyer DB. The human vertebral column at the end of the embryonic period proper. 2 . The occipitocervical re-
gion.J Anat.1983;136(Pt1):181-95. [PubMed: 6833119]. [PubMed Central: PMC1171939].

10. O'Rahilly R, Muller F, Meyer DB. The human vertebral column at the end of the embryonic period proper. 3. The thoracicolumbar region. J Anat. 1990;168:81-93. [PubMed: 2323997]. [PubMed Central: PMC1256892].

11. O'Rahilly R, Muller F, Meyer DB. The human vertebral column at the end of the embryonic period proper. 4 . The sacrococcygeal region. J Anat. 1990;168:95-111. [PubMed: 2182589]. [PubMed Central: PMC1256893].

12. Panattoni GL, Todros T. The fetal development of the human vertebral column as imaged by ultrasound. New Trend Gynaecol Obst. 1986;2:16578.

13. Noback CR, Robertson GG. Sequences of appearance of ossification centers in the human skeleton during the first five prenatal months. Am J Anat. 1951;89(1):1-28. doi: 10.1002/aja.1000890102. [PubMed 14877766].

14. Ulm MR, Kratochwil A, Oberhuemer U, Ulm B, Blaicher W, Bernaschek G. Ultrasound evaluation of fetal spine length between 14 and 24 weeks of gestation. Prenat Diagn. 1999;19(7):637-41. doi: 10.1002/(SICI)1097-0223(199907)19:7<637::AID-PD611>3.0.CO;2-X. [PubMed: 10419611].

15. Flecker H. Time of appearance and fusion of ossification centers as observed by roentgenographic methods. AJR. 1942;47:97-159. 